\title{
BMJ Open Web-based physical activity intervention for people with progressive multiple sclerosis: application of consensus-based intervention development guidance
}

\author{
Monica Busse (D) , ${ }^{1}$ Julie Latchem-Hastings, ${ }^{1}$ Kate Button, ${ }^{2}$ Vince Poile (D) , ${ }^{1}$ \\ Freya Davies (1) , ${ }^{3}$ Rhian O' Halloran, ${ }^{4}$ Barbara Stensland, ${ }^{1}$ Emma Tallantyre, ${ }^{5}$ \\ Rachel Lowe, ${ }^{1}$ Fiona Wood (1) , ${ }^{3}$ Helen Dawes, ${ }^{6}$ Adrian Edwards, ${ }^{3}$ Fiona Jones ${ }^{7}$
}

To cite: Busse M, LatchemHastings J, Button K, et al. Web-based physical activity intervention for people with progressive multiple sclerosis: application of consensus-based intervention development guidance. BMJ Open 2021;11:e045378. doi:10.1136/ bmjopen-2020-045378

- Prepublication history and additional material for this paper is available online. To view these files, please visit the journal online (http://dx.doi.org/10. 1136/bmjopen-2020-045378).

$\mathrm{MB}$ and $\mathrm{JL}-\mathrm{H}$ contributed equally.

Received 30 September 2020 Revised 15 February 2021 Accepted 07 March 2021

Check for updates

(C) Author(s) (or their employer(s)) 2021. Re-use permitted under CC BY-NC. No commercial re-use. See rights and permissions. Published by BMJ.

For numbered affiliations see end of article.

Correspondence to Professor Monica Busse; busseme@cardiff.ac.uk

\section{ABSTRACT}

Objectives People with progressive multiple sclerosis (PwPMS) report that they recognise the benefits of activity on their physical and psychological health but need support to achieve their physical activity goals. We aimed to systematically develop a theoretically informed intervention that would enable PwPMS to more readily engage in regular physical activity.

Design We used an intervention mapping approach to inform intervention development.

Setting We conducted semistructured interviews with PwPMS and their families/carers and physiotherapists recruited from secondary care settings.

Participants Fourteen PwPMS with an Expanded Disability Status Scale score of between 6 and 8 and 7 of their families/carers and 13 physiotherapists and 1 physiotherapy technician participated.

Results Interview data suggested that the development of supportive coaching relationships with physiotherapists could promote the ability of PwPMS to achieve a desirable and achievable physical activity plan. These interview data informed the prototype 'Lifestyle Exercise and Activity Package for Multiple Sclerosis' (LEAP-MS) consisting of a secure multiuser web-based platform (with an education and activity suite, interactive components enabling selection of exercises, goal setting and activity logging), up to six flexible face-to-face or web-based physiotherapy coaching sessions and remote support via an embedded web-based messaging function that all together draw on specific theory-based methods to achieve physical activity behaviour change, namely active learning, reinforcement, modelling, feedback, facilitation, goal setting and guided practice. Implementation is within a multiuser platform accessible to participants, trained physiotherapists and researchers.

Conclusions We have followed an inclusive, systematic and transparent process to develop the LEAP-MS intervention that enables detailed description of components, context and guiding principles to inform ongoing evaluation. Importantly, PwPMS expressed the need for autonomy in developing physical activity plans. This has been achieved through the embedding of self-

\section{Strengths and limitations of this study}

- We have followed a systematic process that adheres to consensus-based intervention development guidance.

- Involvement of key stakeholders was central throughout to make the resultant intervention grounded in the particular lived experiences and needs of this underserved patient group.

- Physiotherapy training to use technology in remote consultations and provide self-management support was developed as part of the implementation planning process.

- Members of the patient and public involvement steering committee reviewed the prototype intervention and volunteered to undertake initial feasibility testing to refine the implementation process but further and more extensive user testing would have been preferable.

- Stakeholders were identified from across South Wales and thus perspectives were limited to one locality.

management principles in the design and delivery of the LEAP-MS intervention.

\section{INTRODUCTION/BACKGROUND}

Multiple sclerosis (MS) is the most common disabling neurological disease among young adults in the developed world ${ }^{1}$ with a wide range of associated symptoms including motor, sensory, visual and autonomic impairments. ${ }^{1}$ It affects an estimated 107000 people in the UK. ${ }^{2}$ These individuals are often high users of health and social care services and self-report low health-related quality-oflife. ${ }^{34}$ Around $10 \%$ of patients are initially diagnosed with progressive MS and many with an initial relapsing remitting course will 
develop progressive MS and advancing disability over time. $^{15}$

The benefits of supervised exercise in MS are well established such that physical activity interventions have become a cornerstone of MS rehabilitation with physical, psychological and social domain benefits. ${ }^{67}$ Regular physical activity can provide a sense of hope and purpose ${ }^{89}$ and people with MS, including those with progressive MS value and perceive benefit from keeping physically active and moving. ${ }^{10}{ }^{11}$ However, in more recent years, there has been a shift in thinking away from 'exercise training for fitness' towards 'physical activity for health'. ${ }^{12}$ To this end, explicitly supporting people with MS with the skills to select or alter their activities and individualise to their preferences, needs, time constraints and values is more likely to achieve a sustained change in behaviour. ${ }^{13}$ Indeed, building self-efficacy through goal setting and mastery along with coaching-style communication and self-monitoring are now well established as critically important determinants of sustained behaviours. ${ }^{7}$

A systematic review (including studies published up until 30 April 2015) identified 19 reports of behavioural change interventions aiming to increase activity and participation in people with MS. Interventions typically incorporated goal setting, barrier identification and information provision and were delivered either face to face, using tele-medicine technology or through unsupervised home-based exercise programmes with written material as support. They were effective in increasing physical activity over the short term, but had no effect on the physical components of quality of life or fatigue and initial increases in physical activity were not sustained in the longer term. ${ }^{14}$ Other researchers have previously developed interventions to promote physical activity in MS using a dedicated website and video chats with a coach. ${ }^{1516}$ However, as with most MS physical activity research to date, ${ }^{6710111718}$ studies have exclusively focused on patients who are ambulatory, despite non-ambulatory people with MS, with more advanced disease being most at risk of becoming inactive.

Seeking sustainable, cost-effective interventions that promote physical activity behaviours for all, in particular those who struggle with higher levels of disability, is clearly a priority. Furthermore providing clarity on the ways in which physiotherapists are best able to contribute to self-management support for physical activity for people with progressive MS (PwPMS), was highlighted during a research priority setting exercise run by the James Lind Alliance in partnership with the MS Society. Thus, we aimed to develop a theoretically informed physiotherapy intervention, the 'Lifestyle Exercise and Activity Package for Multiple Sclerosis' (LEAP-MS), that would to enable PwPMS to more readily engage in regular physical activity both in and out of their homes.

We used intervention mapping, ${ }^{19}$ a systematic approach to intervention development, that incorporates theory and evidence-based planning for behavioural change and acknowledges individuals' behaviour as being dependent on their environment and social context. It has been applied in a range of behaviour change contexts and settings (both clinical and public health) including physical activity promotion in older age individuals, ${ }^{20}$ selfmanagement interventions for rheumatoid arthritis, ${ }^{21}{ }^{22}$ and for low back pain ${ }^{23}$ and theory-based interventions to promote physical activity in preschool and school age children. $^{2425}$

The first step of the intervention development process is the needs assessment which aims to identify areas for change, associated behavioural and environmental factors (barriers) that together inform the definition of intervention context and goals. Here, we report on the initial needs assessment and the subsequent development of LEAP-MS.

\section{METHODS}

We used semistructured interviews to explore (1) the experiences of PwPMS (with significant disability limiting their walking and ability to participate in activities of daily living) and their families/carers in accessing and doing physical activity and (2) the perceptions, experiences and training needs of physiotherapists in supporting PwPMS to be physically active.

PwPMS were identified via a tertiary MS centre database, which held records of all persons with MS in the region that had given permission to be contacted about research projects applicable to them. Purposive sampling to ensure a representative balance of gender, age and Expanded Disability Status Scale (EDSS) ${ }^{26}$ score (with EDSS range between six and eight) was employed. Family members/carers were also invited to participate. Written informed consent was obtained for all research interviews apart from one interview where the participant was unable to hold a pen. In this case consent was verbal and witnessed. Physiotherapists and physiotherapy technicians were recruited via three participating University Health Boards.

Face-to-face interviews were conducted by a postdoctoral neurological physiotherapist with experience working with patients with MS and conducting and tailoring semi-structured interviews with people with a wide range of neurological deficits. Interviews explored past and present physical activity, including facilitators and barriers, support from physiotherapists (or not), experiences/understanding of 'self-management' and their ideas for personalising an invention resource, (see Interview Topic Guide; online supplemental file 1). Interviews with physiotherapists focused on their experiences of supporting PwPMS to be physically active, their understanding of self-management principles and the application of these in everyday practice.

Interviews were audiorecorded, transcribed verbatim and analysed thematically. ${ }^{27}$ This involved familiarisation with the data by rereading and annotating transcripts. The data were then systematically searched for recurring themes. Crucially, the researcher did not just look 
at recurring themes but also views that were unusual or contradictory. Analytic notes were made and labels (codes) were applied to sections of text. Codes were then grouped into categories of shared meaning. The strength of each initial category was considered, and key themes established through an inductive, latent thematic analysis. Categorical groupings were restructured as the data were revisited and themes which best captured the essence of the data formed. Once the final themes had been established, the data were revisited to ensure that all manifestations of each theme in the dataset had been accounted for. ${ }^{28}$

Six physiotherapists, 10 PwPMS and 5 family members attended a stakeholder event to contribute to step 4 of the intervention mapping process. They discussed (1) key barriers to physical activity and (2) possible solutions. These individuals were identified through advertising posters in MS specialist clinic areas and a local social media campaign with the support of the local MS Society and our public and patient involvement (PPI) group. Those present were asked to think about physical activities that they could do and those which they could envisage being of interest to others.

In one of four mini workshops attendees explored their readiness (or not) to access and use available technologies.

\section{Patient and public involvement}

The research question emerged as a result of a James Lind Alliance Priority Setting Exercise. Patients and the public were involved in the design (interview and stakeholder event planning), conduct (delivering the stakeholder event and interpretation of the interview data), reporting of this research (developing a communication and producing video summaries of the findings) through an established PPI group. The chair of the PPI group was a member of the study management group attending regular management group meetings. The PPI group remain involved in ongoing dissemination plans for this research.

\section{RESULTS}

The interview data (needs assessment) guided each subsequent intervention development step namely: (1) specification of a logic model of change based on anticipated intervention outcomes; performance objectives and behavioural determinants; (2) selection of applicable theory-based intervention methods and practical applications relevant to the determinants specified in (1); (3) development of a prototype intervention based on (2) to (3) as well as the aforementioned stakeholder event; (4) development of an adoption and implementation plan relevant to the specific intervention contexts and (5) design of an evaluation plan to assess process and future effectiveness. Main outcomes from each step of the process are depicted in figure 1.

\section{Step 1: needs assessment}

Fourteen PwPMS with an EDSS score of between 6 and 8 and 7 of their families/carers participated in face-to-face

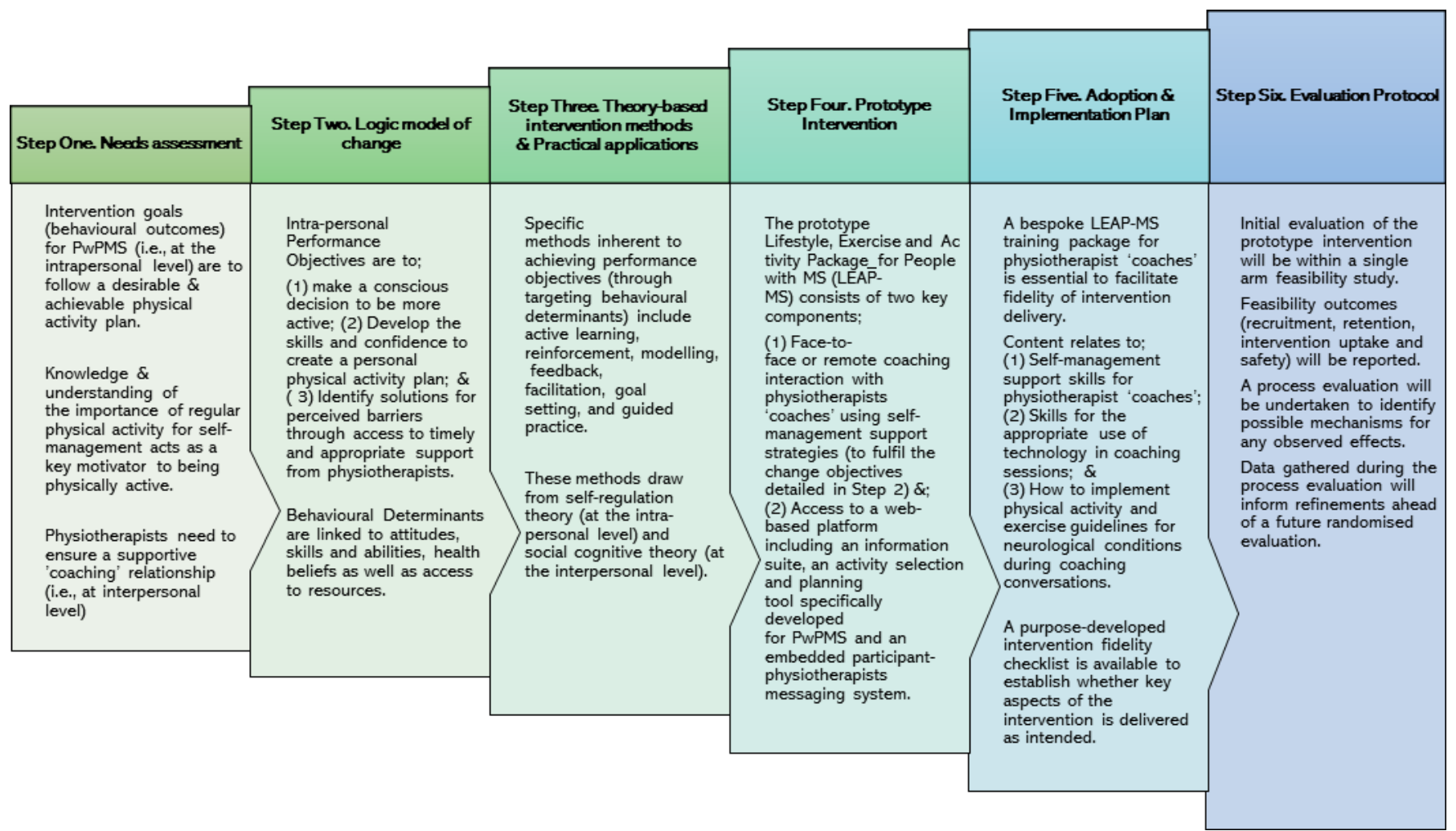

Figure 1 Main outcomes at each step of the six step intervention mapping process. PwPMS, People with progressive Multiple Sclerosis. 
interviews. Thirteen physiotherapists and one physiotherapy technician participated in interviews. We identified eight themes from interviews with PwPMS (and families/carers) and three themes from physiotherapist interviews that informed the intervention goals, namely that the PwPMS would develop a desirable and achievable physical activity plan. Critically this would be achieved through the development of a supportive coaching relationship with their physiotherapist.

Table 1 demonstrates the key themes and how they were associated with the overarching LEAP-MS intervention goals (behavioural outcomes), the related change and performance objectives and relevant behavioural determinants at both the intrapersonal level (ie, for the person with PwPMS) and the intrapersonal (ie, the physiotherapist). Practical implications for the intervention development are also detailed in table 1 .

\section{Step 2: developing a logic model of change}

We produced a logic model of change (figure 2) which linked performance objectives to behavioural determinants that would lead to the proposed intervention outcomes over the short and medium term. Intrapersonal (for the PwPMS) performance objectives focused around three main areas-first, making a conscious decision to be more active; second, developing the skills and confidence to create a personal physical activity plan, which would include identifying suitable physical activities, ${ }^{29}$ weighing up the risks and benefits of each activity in relation to health, and to be confident in trying new things and third-identifying solutions for perceived barriers through access to timely and appropriate support from physiotherapists. A key dependency was that the physiotherapists themselves recognised the importance of personalised and tailored self-management support for physical activity and that they had the knowledge, confidence and skills to do so.

\section{Step 3: selection of applicable theory-based intervention methods and applications}

Recognising the need for specific methods to achieve physical activity behaviour change, for example, active learning, reinforcement, modelling, feedback, facilitation, goal setting and guided practice, ${ }^{30}$ we drew from two underlying programme theories: social cognitive theory and self-regulation theory. Social cognitive theory is a widely adopted theoretical framework used in physical activity interventions. ${ }^{31}{ }^{32}$ Within social cognitive theory, behaviour is an outcome of the interaction of personal, social and physical factors within the environment and knowledge of self that will influence health beliefs and behaviours. The key construct within social cognitive theory is that of self-efficacy, defined as 'a belief about individual capability to perform a certain behaviour'. ${ }^{31}$ Other critical constructs are in relation to exploring health beliefs, outcome expectations, barriers and facilitators and using knowledge of self in goal setting. ${ }^{33-36}$ Specific methods inherent in social cognitive theory-based interventions are goal mastery, active learning, reinforcement, modelling, feedback, facilitation and guided practice. $^{37}$

Self-regulation theory argues that illness representations, beliefs and social and cultural contexts drive behavioural and emotional responses. ${ }^{38}$ Representations of the illness are personally evaluated, and individuals will typically respond to awareness of an issue by developing an action plan achieved through guided practice and goal setting. ${ }^{37}$ Online supplemental tables 1 and 2 depict the matrix of performance objectives, behavioural determinants and change objectives with associated theory-based methods, parameters and practical applications at the intrapersonal and interpersonal levels.

\section{Step 4: designing and producing the intervention}

The way in which PwPMS wanted to engage with physiotherapists was a critical factor in intervention design. Discussions at our stakeholder event highlighted that physiotherapy input in supporting physical activity engagement was warranted but ownership (autonomy) was critical to ongoing engagement. The presence of physiotherapists was only useful if they could recognise specific contexts and work with PwPMS to address their concerns. Flexible needs-driven communication with physiotherapists was preferred; those who saw physiotherapists as key motivators to their level of activity preferred more regular, scheduled contact.

Participants in our stakeholder event detailed extensive variety in activities which interested them indicating a requirement for a vast 'library' of activity 'ideas' to meet the needs of a very diverse population. The event confirmed our interview findings that a technological solution would be welcomed but that such a solution would require interactive functionality that could be personalised with text, audio and visual options for providing information, as well as access to remote support from physiotherapists. Many stakeholders had multiple personal technologies that they used daily in their home and most accessed the internet regularly to gain support and information about MS. Attendees were quick to suggest technological solutions to engaging with both activities and therapists and envisaged various features they would like to see in webbased tools-especially for the support of memory and motivation. They also identified that technology offered the potential for personalisation in a way that other methods could not.

The LEAP-MS intervention (www.leapms.org) was thus designed to consist of two key components: (1) face-toface or web-based coaching with physiotherapists using self-management support strategies (to fulfil the change objectives detailed in step 2) and (2) a web-based platform including an information suite, an activity selection and planning tool specifically developed for PwPMS and a participant-physiotherapist messaging system (see figure 3). It was developed as a multiuser system that enables the pairing of MS participant and physiotherapist accounts. It promotes self-management and 
Table 1 Themes and key messages identified from interviews with PwPMS (and families/carers) and physiotherapists as well as associated LEAP-MS intervention goals, change objectives and practical considerations

\begin{tabular}{ll}
\hline Theme & Key messages \\
\hline $\begin{array}{l}\text { Experience of } \\
\text { information at } \\
\text { point of diagnosis }\end{array}$ & $\begin{array}{l}\text { How diagnosis was delivered and remembered by } \\
\text { the participant was often influential in shaping their } \\
\text { initial attitude towards their condition and could } \\
\text { also impact on their long-term attitude towards } \\
\text { physical activity. }\end{array}$ \\
$\begin{array}{l}\text { Relationship with } \\
\text { physical activity } \\
\text { and exercise pre- } \\
\text { MS and post-MS }\end{array}$ & $\begin{array}{l}\text { The relationships and attitudes PwPMS had with } \\
\text { physical activity, sport and exercise before their MS } \\
\text { diagnosis could impact on their attitudes towards } \\
\text { physical activity following diagnosis. }\end{array}$ \\
impact of physical \\
activity on MS \\
symptoms
\end{tabular} $\begin{aligned} & \begin{array}{l}\text { Those who were clear that physical activity helped } \\
\text { them manage their symptoms were more engaged } \\
\text { with physical activity than those who could not see } \\
\text { how it could help them. }\end{array} \\
& \text { Motivation }\end{aligned} \begin{aligned} & \text { What did and did not motivate PwPMS to be } \\
& \text { physically active was highly individual-but } \\
& \text { included the ability to recognise how physical } \\
& \text { activity could help manage MS symptoms and the } \\
& \text { input of healthcare professions and peers. }\end{aligned}$

Fear, falls and The single biggest barriers to activity for people fatigue with progressive MS were either fatigue or a fear of falling.

Therapies and interactions with healthcare professionals

Reliance and risk Most were happy to take measured risks but took support from (and spoke about the importance of support given by) partners and family members to help them mitigate risks as much as possible.
Finance and facilities
Past and present interactions with physiotherapists and other healthcare professionals also played a part in attitudes towards physical activity.

\section{Intervention goals}

(behavioural outcomes)

\section{Change objectives}

Person with MS

follows a desirable

and achievable

physical activity plan
Decide to be more

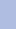

active with MS

Identify solutions for Recognising the individuality of 'motivation'-we perceived barriers included a range of functions and features in the LEAP-MS intervention including goal setting and self-progress monitoring, the option of meeting with physiotherapists up to six times and the ability for physiotherapists to view participants plans. This sense of being monitored by healthcare professions was seen as a key motivator for doing regular activities discussed.

We included information and advice about exercise and the management of fatigue in an 'Information Suite'. We carefully selected activities including lots of seated options to enable activity with very low risk for those for whom either fear or risk of falling was high in the web platforms' 'Activities Suite' and ensured key safety information and reminders were populated throughout the platform.

We trained physiotherapists to take a self-management approach to their coaching sessions with participants which focused on enhancing physical activity rather than solving a particular problem or issue. Using self-management and focusing on enhancing wellbeing rather than looking at problems provided the opportunity for the participant-physiotherapy relationship to be re-set.

Create own Recognising the importance of exercising together we personalised physical recommended exercise with others on the LEAP-MS Activity plan platform and for example, included activities which could be carried out with children to provide options for families to be active together.

We provided basic safety information in the 'Information Suite' and through the use of selfmanagement techniques in physiotherapy coaching sessions, supported (and skilled) PwPMS to make their own judgements about safety and mitigating risks.

We ensured that LEAP-MS content and/or activities suggested were primarily: (1) free, (2) could be done at home or in open public spaces and/or (3) did not rely on expensive equipment.

LEAP-MS provided alternative ways to meet with physiotherapists (eg, over zoom). technology for a range of social and work purposes. While none were using these devices to support physical activity, the potential of online engagement to support physical activity was largely welcomed.

Physiotherapists Physiotherapists recognised the importance as experts of physical activity in the management of MS symptoms, but were less confident in the application of exercise principles specific to the needs of people with long term neurological conditions.

\section{Physiotherapist} efficiently supports people with MS to engage in a desirable and achievable physical activity plan
We developed a training package for physiotherapists which included asynchronous self-study of exercise principles for people with long term neurological conditions.

\section{Demonstrate}

confidence to develop a shared approach to exercise prescription, knowledge and skills

to shape programmes to fit with individual needs

Endorse creative and individualised physical activity planning in LEAP-MS consultations

Focus on individual Physiotherapists explained that there was little problems rather opportunity for physical activity', general fitness or than on physical exercise to be a central focus of a session due to activity service structure and provision.

Self-management Self-management as an approach was well approaches to accepted by those we interviewed but the level of physical activity skill and experience of using this approach varied significantly within our cohort. 
Individual (Intrapersonal level)

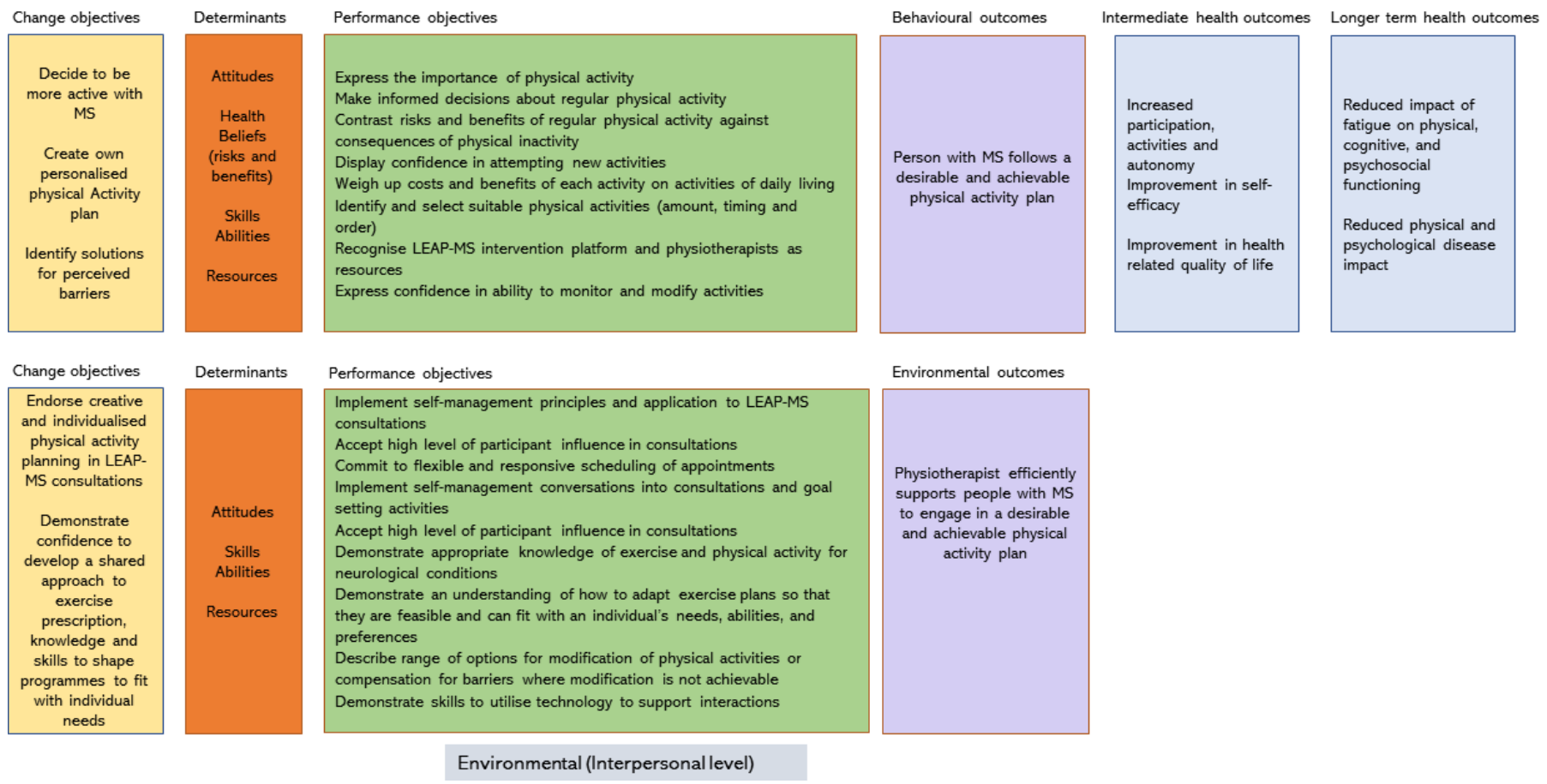

Figure 2 Logic model of change for the intervention detailing change objectives determinants, performance objectives and proposed behavioural (intrapersonal) and environmental (interpersonal) outcomes leading to intermediate and longer-term health outcomes for the person with multiple sclerosis (MS). LEAP-MS, Lifestyle Exercise and Activity Package for Multiple Sclerosis.

autonomous behaviour supported by physiotherapist as needed. In-platform messaging enables the physiotherapist to see and discuss participant choices, aims and progress building on a previous successfully tested multiuser system $\left(\right.$ TRAK $^{39}{ }^{40}$ ).

The physical activity coaching approach is a development of an existing work book-based physical activity coaching intervention for people with Huntington's disease. $^{41}$ It promotes self-management skills through the use of coaching techniques and strategies as part of a Bridges self-management approach. ${ }^{42}$ In coaching sessions, PwPMS will learn how to navigate the LEAP-MS website, choose personal activities, set goals and log weekly activity. Physiotherapists will draw on their professional knowledge and skills of safe, appropriate physical activity and exercise specific to MS both verbally (face to face or remote) and through directing participants to the LEAP-MS web-based platform. Depending on needs and areas of importance identified by the PwPMS, they will encourage self-management approached through the discussion of strategies to anticipate and overcome barriers to physical activity and explore options for selfmonitoring (including activity trackers and logs). They may also share examples of other PwPMS who have experienced similar challenges when initiating a new exercise programme-with discussion of how they overcame these, thus using modelling as a potential source of self-efficacy. After the initial session, physiotherapists will review any progress, safety concerns and progression of exercise as requested or initiated by the PwPMS and provide appropriate feedback for activity selection, attempts and engagement in regular physical activity. PwPMS can also use the inbuilt messaging function to communicate with their paired physiotherapist to seek additional support if needed.

\section{Step 5: planning for adoption and implementation}

A bespoke LEAP-MS training package ${ }^{43}$ which ensures skill development in provision of self-management support skills for physiotherapist ${ }^{42}$ alongside the appropriate use of technology in coaching sessions and application of physical activity and exercise guidelines for neurological conditions has been developed. ${ }^{44}$ The initial content was achieved through the conduct of a 2 day interactive training session, attended by physiotherapists likely to deliver the intervention in the subsequent evaluation, underpinned by Bridges Self-Management principles and delivered by Bridges Social Enterprise (http:// www.bridgesselfmanagement.org.uk/). This workshop was video recorded and prepared for use as a final online training package accessed via the LEAP-MS multimedia online learning resource. Given the emergent challenges in rehabilitation service delivery during the COVID-19 pandemic, ${ }^{45-47}$ and the anticipated move to greater use of remote intervention delivery, further resources (https://www.bridgesselfmanagement.org.uk/covid-19resources/) to help structure remote interactions were made available as part of the final training package so as to ensure standardisation of coaching interactions 


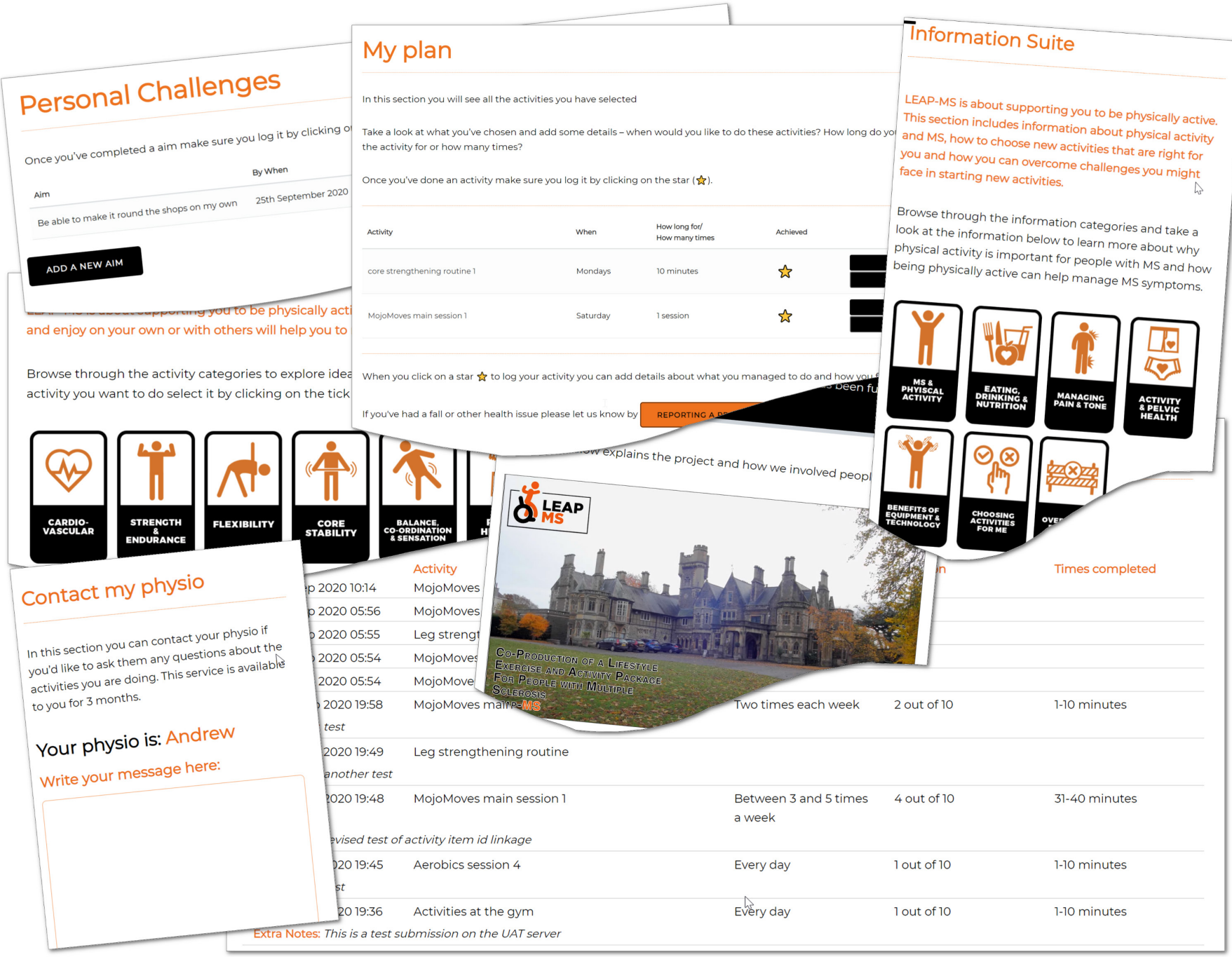

Figure 3 The web-based platform which includes multimedia education about being active with MS, tailored physical activity ideas and interactive functions enabling the development of personalised activity programmes, goal setting and activity logs. Unlike other physiotherapy-based web-based activity platforms for other conditions or general education platforms, the platform has a paired account function in which people with MS can be paired with their physiotherapist. Critically, rather than the physiotherapist selecting and prescribing activities, the person with MS has complete choice and control of this process. MS, multiple sclerosis.

regardless of mode of delivery (online or face to face in the home).

The key knowledge and skills required by physiotherapists to deliver the intervention informed specific training and the onwards planning for fidelity evaluation were captured in a purpose-developed fidelity checklist (online supplemental table 3). They include understanding of exercise principles and physical activity specifically for neurological conditions and those needed to facilitate the central approach of selfmanagement, the ability to implement coaching conversations underpinned by self-management principles and language, having an understanding of how to adapt/ modify exercise plans so that they are feasible and can fit with an individual's needs, and abilities, favourable attitudes and the technical skills to support PwPMS to use web-based tools.

\section{Step 6: evaluation plan}

In line with the Medical Research Council Framework for Development and Evaluation of complex interventions ${ }^{48}{ }^{49}$ LEAP-MS will be evaluated within a single arm feasibility study with embedded process evaluation. ${ }^{43}$ After registration and initial eligible screening, 21 participants will be required to complete baseline self-report measures after which they will be able to access the intervention for an initial 3-month period. During this period, participants are given the option to request up to five further home-based or online physiotherapy coaching sessions as found acceptable in previous physical activity interventions using components of this intervention. ${ }^{41}$

Follow-up questionnaires and semistructured interviews will be administered 3 months after baseline with participants and intervention physiotherapists. The LEAP-MS platform will be available to participants for a further 
3 months. Use of the LEAP-MS platform will be tracked during the full 6-month period and final follow-up will be conducted 6 months after baseline. Given the detailed development process followed it will be possible for the intervention to be fully described in line with the 'Template for Intervention Description and Repli-cation' checklist. ${ }^{50}$ Feasibility outcomes (recruitment, retention, intervention fidelity, uptake (website usage statistics) and safety) will be reported and the process evaluation will explore participant experiences of the intervention and explore possible mechanisms for any observed effects.

\section{DISCUSSION}

Remote technologies to support the delivery of medical, mental health and rehabilitation services have been documented for many years and, with the ever-growing refinement and accessibility of technologies within the home, so has the interest of service users in remote delivery of health-based services. ${ }^{51}$ Telephone and webbased consultations for physiotherapy or musculoskeletal based assessment/outpatient triage has been in use for several decades, ${ }^{52}$ and the use of 'wearables'-personal activity devices to monitor and motivate activity-account for much contemporary digital discussion in therapeutic literature. ${ }^{53-55}$

A vast array of digital technologies has been harnessed to support all aspects of MS care. ${ }^{56}$ Examples include those which focus particularly on physical activity via web-based units of self-directed learning combined with exercise programmes prescribed by a physiotherapist and a texting or email service, ${ }^{57}$ in-clinic prescribed exercise programmes combined with home-based automated prompt systems delivering reminders, ${ }^{58}$ web-displayed physiotherapy-prescribed exercise routines alongside telephone calls or web-based asynchronous communication with physiotherapists. ${ }^{59-61}$

None of these interventions however focus on people with high levels of disability and impairment and they do not combine real-time web conferencing to support patient-physiotherapist communication. Within this context, the LEAP-MS is unique in providing a tailored physical activity self-management support intervention for PwPMS with severe disability. The strong expression from PwPMS about autonomy and the ethos and embedding of self-management principles that was evident throughout this intervention mapping process was central to design and delivery of the LEAP-MS intervention. The resultant LEAP-MS intervention focuses on coaching PwPMS to consider and plan their own opportunities for physical activity through eliciting, acknowledging, and discussing participants' perceptions and experiences of physical activity. ${ }^{33-36}$ It seeks to ensure PwPMS have control over when and how they access physiotherapist input including home-based, face-to-face or on-line (video based) coaching sessions. These coaching strategies directly support self-management through promoting goal setting and development of a personalised activity plan $^{41}$ as part of a Bridges self-management approach. ${ }^{42}$ In this, key sources of self-efficacy, goal mastery and modelling are integral to coaching interactions, as both mediator of change and an anticipated outcome.

We followed the GUIDance for the rEporting of intervention Development framework to provide a comprehensive description of each step of the intervention development process. ${ }^{62}$ The context, purpose and target population were clearly defined and we used the six step intervention mapping process ${ }^{63}$ to develop a theoretically informed intervention. We ensured participation of all stakeholders throughout the process both through the conduct of interviews to inform the needs assessment and at the intervention design stage when we hosted a stakeholder event with a view to ensuring acceptability and relevance of our outputs. Interview participants and stakeholders were identified from across South Wales. Three members of our PPI steering committee reviewed the prototype intervention and engaged in initial feasibility testing to help the team refine implementation plans although we have yet to establish optimal delivery mode (face to face or remote are both options) and dose (participants can elect to receive up to six coaching sessions) and we cannot be sure that we have captured relevant content across all stages of progressive MS. While the ascertainment of views were limited to those in one single geographical area, the views expressed by our participants were well aligned to that reported in the literature in terms of barriers and facilitators of physical activity in PwPMS. ${ }^{17} 2936$ Future associated studies will need to incorporate views of PwPMS from a broader spread of localities with specific reference to the role of physiotherapist coaches in providing self-management support for physical activity in PwPMS.

LEAP-MS is a bespoke intervention that addresses the fundamental, individual, and relational barriers to maintaining meaningful activity that are not routinely considered in directed and structured physiotherapy interventions. It is now ready for initial evaluation in a single-arm feasibility and acceptability study with embedded process evaluation (https://clinicaltrials.gov/ct2/show/NCT03951181). It is our view that our ongoing emphasis on codesign will ensure intervention acceptability and adherence and this initial evaluation will seek to provide robust user experience data which will inform further intervention refinement ahead of full-scale evaluation.

\section{Author affiliations \\ ${ }^{1}$ Centre for Trials Research, Cardiff University, Cardiff, UK \\ ${ }^{2}$ School of Healthcare Sciences, Cardiff University, Cardiff, UK \\ ${ }^{3}$ Division of Population Medicine, Cardiff University, Cardiff, UK \\ ${ }^{4}$ Cardiff and Vale UHB, Cardiff, UK \\ ${ }^{5}$ Division of Psychological Medicine and Clinical Neuroscience, Cardiff University, Cardiff, UK \\ ${ }^{6}$ Department of Sport and Health Sciences, Oxford Brookes University, Oxford, UK ${ }^{7}$ Centre for Health and Social Care Research, St George's University of London and Kingston University, London, UK}

Acknowledgements We would like to thank our public and patient involvement (PPI) steering group made up of PwPMS, their family and charity stakeholders who collaborated with us from the start to the end of the project. We would also 
like to acknowledge the critical input from Janine Bates, Charlotte Scoble, Christy Barlow and Elizabeth Randell in the initial studies and activities that informed the development of the ongoing study evaluating LEAP-MS. Further acknowledgement is to Fizzi Productions for their creative input to the project. Centre for Trials Research and PRIME Centre Wales, Cardiff University receives infrastructure support from Health and Care Research Wales. Professor Helen Dawes is supported by the Elizabeth Casson Trust and the NIHR Oxford Health Biomedical Research Centre.

Contributors MB conception, design, implementation, drafting and finalising manuscript. JL-H conception, design, implementation, drafting and finalising manuscript. KB conception, design, implementation, revising manuscript for intellectual content and final approval. VP design, implementation, revising manuscript for intellectual content and final approval. FD conception, design, implementation, revising manuscript for intellectual content and final approval. RL conception, design, implementation, revising manuscript for intellectual content and final approval of manuscript. RO conception, design, implementation, revising manuscript for intellectual content and finalising manuscript. BS conception, design, implementation, revising manuscript for intellectual content and finalising manuscript. ET conception, design, implementation, revising manuscript for intellectual content and finalising manuscript. FW conception, design, implementation, revising manuscript for intellectual content and final approval. HD conception, design, implementation, revising manuscript for intellectual content and final approval. AE conception, design, implementation, revising manuscript for intellectual content and final approval. FJ conception, design, implementation, revising manuscript for intellectual content and final approval.

Funding This work was supported by the Multiple Sclerosis Society grant number 81.

Disclaimer The views expressed are those of the authors and not necessarily those of the NHS, the NIHR or the Department of Health.

Competing interests None declared.

Patient consent for publication Not required.

Ethics approval Ethical approval for all research activities underpinning this intervention development study conducted over a 24-month period (July 2018 to July 2020) was granted by Wales Research Ethics Committee 3.

Provenance and peer review Not commissioned; externally peer reviewed.

Data availability statement Deidentified interview data are available from the Centre for Trials Research on reasonable request (https://www.cardiff.ac.uk/centrefor-trials-research/about-us/data-requests).

Supplemental material This content has been supplied by the author(s). It has not been vetted by BMJ Publishing Group Limited (BMJ) and may not have been peer-reviewed. Any opinions or recommendations discussed are solely those of the author(s) and are not endorsed by BMJ. BMJ disclaims all liability and responsibility arising from any reliance placed on the content. Where the content includes any translated material, BMJ does not warrant the accuracy and reliability of the translations (including but not limited to local regulations, clinical guidelines, terminology, drug names and drug dosages), and is not responsible for any error and/or omissions arising from translation and adaptation or otherwise.

Open access This is an open access article distributed in accordance with the Creative Commons Attribution Non Commercial (CC BY-NC 4.0) license, which permits others to distribute, remix, adapt, build upon this work non-commercially, and license their derivative works on different terms, provided the original work is properly cited, appropriate credit is given, any changes made indicated, and the use is non-commercial. See: http://creativecommons.org/licenses/by-nc/4.0/.

\section{ORCID iDs}

Monica Busse http://orcid.org/0000-0002-5331-5909

Vince Poile http://orcid.org/0000-0002-5770-5584

Freya Davies http://orcid.org/0000-0002-6956-1100

Fiona Wood http://orcid.org/0000-0001-7397-4074

\section{REFERENCES}

1 Murray TJ. Diagnosis and treatment of multiple sclerosis. BMJ 2006;332:525-7.

2 Public Health England. Multiple sclerosis: prevalence, incidence and smoking status, 2020. Available: https://www.gov.uk/government/ publications/multiple-sclerosis-prevalence-incidence-and-smokingstatus
3 McCrone P, Heslin M, Knapp M, et al. Multiple sclerosis in the UK: service use, costs, quality of life and disability. Pharmacoeconomics 2008;26:847-60.

4 Kobelt G, Thompson A, Berg J, et al. New insights into the burden and costs of multiple sclerosis in Europe. Mult Scler 2017;23:1123-36.

5 Harding KE, Wardle M, Moore P, et al. Modelling the natural history of primary progressive multiple sclerosis. J Neurol Neurosurg Psychiatry 2015;86:13-19.

6 Dalgas U, Langeskov-Christensen M, Stenager E, et al. Exercise as medicine in multiple Sclerosis-Time for a paradigm shift: preventive, symptomatic, and disease-modifying aspects and perspectives. Curr Neurol Neurosci Rep 2019;19:88.

7 Backus D. Increasing physical activity and participation in people with multiple sclerosis: a review. Arch Phys Med Rehabil 2016;97:S210-7.

8 Soundy A, Benson J, Dawes $\mathrm{H}$, et al. Understanding hope in patients with multiple sclerosis. Physiotherapy 2012;98:344-50.

9 Feinstein A, Freeman J, Lo AC. Treatment of progressive multiple sclerosis: what works, what does not, and what is needed. Lancet Neurol 2015;14:194-207.

10 Motl RW, Dlugonski D. Increasing physical activity in multiple sclerosis using a behavioral intervention. Behav Med 2011;37:125-31.

11 Dlugonski D, Motl RW, Mohr DC, et al. Internet-Delivered behavioral intervention to increase physical activity in persons with multiple sclerosis: sustainability and secondary outcomes. Psychol Health Med 2012;17:636-51.

12 Motl RW. Lifestyle physical activity in persons with multiple sclerosis: the new kid on the MS block. Mult Scler 2014;20:1025-9.

13 The Health Foundation Behavioural Insights Team. Making the change. behavioural factors in person- and community-centred approaches for health and wellbeing, 2020. Available: https://www. health.org.uk/publications/making-the-change

14 Sangelaji BSmith CM, Smith CM, Paul L, et al. The effectiveness of behaviour change interventions to increase physical activity participation in people with multiple sclerosis: a systematic review and meta-analysis. Clin Rehabil 2016;30:559-76.

15 Silveira SL, McCroskey J, Wingo BC, et al. eHealth-Based behavioral intervention for increasing physical activity in persons with multiple sclerosis: fidelity protocol for a randomized controlled trial. JMIR Res Protoc 2019;8:e12319.

16 Motl RW, Sandroff BM, Wingo BC, et al. Phase-lii, randomized controlled trial of the behavioral intervention for increasing physical activity in multiple sclerosis: project BIPAMS. Contemp Clin Trials 2018;71:154-61.

17 Marck $\mathrm{CH}$, Hadgkiss EJ, Weiland TJ, et al. Physical activity and associated levels of disability and quality of life in people with multiple sclerosis: a large international survey. BMC Neurol 2014;14:143.

18 Hayes S, Uszynski MK, Motl RW, et al. Randomised controlled pilot trial of an exercise plus behaviour change intervention in people with multiple sclerosis: the step it up study. BMJ Open 2017;7:e016336.

19 Kok G, Peters LWH, Ruiter RAC. Planning theory- and evidencebased behavior change interventions: a conceptual review of the intervention mapping protocol. Psicol. Refl. Crít. 2017;30:19.

20 van Stralen MM, Kok G, de Vries $\mathrm{H}$, et al. The active plus protocol: systematic development of two theory- and evidence-based tailored physical activity interventions for the over-fifties. BMC Public Health 2008;8:399.

21 Zuidema RM, van Gaal BGI, van Dulmen S, et al. An online tailored self-management program for patients with rheumatoid arthritis: a developmental study. JMIR Res Protoc 2015;4:e140.

22 Zuidema R, van Dulmen S, Nijhuis-van der Sanden M, et al. Efficacy of a web-based self-management enhancing program for patients with rheumatoid arthritis: explorative randomized controlled trial. $J$ Med Internet Res 2019;21:e12463.

23 Hurley DA, Murphy LC, Hayes D, et al. Using intervention mapping to develop a theory-driven, group-based complex intervention to support self-management of osteoarthritis and low back pain (SOLAS). Implement Sci 2016;11:56.

24 Ten Hoor GA, Kok G, Rutten GM, et al. The Dutch 'Focus on Strength' intervention study protocol: programme design and production, implementation and evaluation plan. BMC Public Health 2016;16:496.

25 De Decker E, De Craemer M, De Bourdeaudhuij I, et al. Using the intervention mapping protocol to reduce European preschoolers' sedentary behavior, an application to the ToyBox-Study. Int J Behav Nutr Phys Act 2014;11:19. 
26 Kurtzke JF. Rating neurologic impairment in multiple sclerosis: an expanded disability status scale (EDSS). Neurology 1983;33:1444-52.

27 Braun V, Clarke V. Using thematic analysis in psychology. Qual Res Psychol 2006;3:77-101.

28 Pope C, Mays N. Qualitative methods in health research. in qualitative research in health care, 3rd edition. Hoboken: Blackwell Publishing Ltd, 2006: 1-11.

29 Kalb R, Brown TR, Coote S, et al. Exercise and lifestyle physical activity recommendations for people with multiple sclerosis throughout the disease course. Mult Scler 2020;26:1459-69.

30 Kok G, Gottlieb NH, Peters G-JY, et al. A taxonomy of behaviour change methods: an intervention mapping approach. Health Psychol Rev 2016;10:297-312.

31 Bandura A. Health promotion by social cognitive means. Health Educ Behav 2004;31:143-64.

32 Bandura A. Social cognitive theory: an Agentic perspective. Annu Rev Psychol 2001;52:1-26.

33 Joseph RP, Ainsworth BE, Mathis L, et al. Utility of social cognitive theory in intervention design for promoting physical activity among African-American women: a qualitative study. Am J Health Behav 2017;41:518-33.

34 Shamizadeh T, Jahangiry L, Sarbakhsh $\mathrm{P}$, et al. Social cognitive theory-based intervention to promote physical activity among prediabetic rural people: a cluster randomized controlled trial. Trials 2019;20:98.

35 Stacey FG, James EL, Chapman K, et al. Social cognitive theory mediators of physical activity in a lifestyle program for cancer survivors and carers: findings from the enrich randomized controlled trial. Int J Behav Nutr Phys Act 2016;13:49.

36 Uszynski MK, Casey B, Hayes S, et al. Social cognitive theory correlates of physical activity in inactive adults with multiple sclerosis. Int J MS Care 2018;20:129-35.

37 Bartholomew LK, Markham C, Ruiter RAC. Behaviour-Oriented theories used in Health Promotion. In: Planning health promotion programs: an intervention mapping approach. 4th. San Francisco: Jossey-Bass, 2016: 57-121.

38 Cameron L, Leventhal $\mathrm{H}$. The self-regulation of health and illness behaviour. Hove: Psychology Press, 2003.

39 Button K, Nicholas K, Busse M, et al. Integrating self-management support for knee injuries into routine clinical practice: TRAK intervention design and delivery. Musculoskeletal Science and Practice 2018;33:53-60.

40 Spasić I, Button K, Divoli A, et al. Trak APP suite: a web-based intervention for delivering standard care for the rehabilitation of knee conditions. JMIR Res Protoc 2015;4:e122.

41 Busse M, Quinn L, Drew C, et al. Physical activity self-management and coaching compared to social interaction in Huntington disease: results from the ENGAGE-HD randomized, controlled pilot feasibility trial. Phys Ther 2017;97:625-39.

42 Jones F, Pöstges H, Brimicombe L. Building Bridges between healthcare professionals, patients and families: A coproduced and integrated approach to self-management support in stroke. NeuroRehabilitation 2016;39:471-80.

43 Latchem-Hastings J, Randall E, Button K. Lifestyle exercise and activity package for people living with progressive multiple sclerosis (LEAP-MS): protocol for a single-arm feasibility study. Pilot and Feasibility Studies 2020 https://www.researchsquare.com/article/rs30578/v1 doi:0.21203/rs.3.rs-30578/v1

44 Collett J, Dawes H, Bateman J. Exercise for Long Term Neurological Conditions: Multiple Sclerosis and Huntington's Disease. In: Clinical Exercise Science. 1st. Oxford: Routledge, 2016: 155-77.
45 Stein J, Visco CJ, Barbuto S. Rehabilitation medicine response to the COVID-19 pandemic. Am J Phys Med Rehabil 2020;99:573-9.

46 Bonavita S, Tedeschi G, Atreja A, et al. Digital triage for people with multiple sclerosis in the age of COVID-19 pandemic. Neurol Sci 2020;41:1007-9.

47 Dantas LO, Barreto RPG, Ferreira CHJ. Digital physical therapy in the COVID-19 pandemic. Braz J Phys Ther 2020;24:381-3.

48 Moore GF, Audrey S, Barker M, et al. Process evaluation of complex interventions: medical Research Council guidance. BMJ 2015;350:h1258.

49 Skivington K, Matthews L, Craig P, et al. Developing and evaluating complex interventions: updating medical Research Council guidance to take account of new methodological and theoretical approaches. The Lancet 2018;392:S2.

50 Hoffmann TC, Glasziou PP, Boutron I, et al. Better reporting of interventions: template for intervention description and replication (TIDieR) checklist and guide. BMJ 2014;348:g1687.

51 Ricker JH, Rosenthal M, Garay E, et al. Telerehabilitation needs: a survey of persons with acquired brain injury. J Head Trauma Rehabil 2002;17:242-50.

52 Russell TG, Blumke R, Richardson B, et al. Telerehabilitation mediated physiotherapy assessment of ankle disorders. Physiother Res Int 2010;15:167-75.

53 Morris J, Jones M, Thompson N, et al. Clinician perspectives on mRehab interventions and technologies for people with disabilities in the United States: a national survey. Int $J$ Environ Res Public Health 2019;16:4220.

54 Jones M, DeRuyter F, Morris J. The digital health revolution and people with disabilities: perspective from the United States. Int $J$ Environ Res Public Health 2020;17:381.

55 Blumenthal J, Wilkinson A, Chignell M, Physiotherapists' CM Physiotherapists' and Physiotherapy Students' Perspectives on the Use of Mobile or Wearable Technology in Their Practice. Physiotherapy Canada 2018;70:251-61.

56 Yeroushalmi S, Maloni H, Costello K, et al. Telemedicine and multiple sclerosis: a comprehensive literature review. J Telemed Telecare 2020;26:400-13.

57 Conroy SS, Zhan M, Culpepper WJ, et al. Self-directed exercise in multiple sclerosis: evaluation of a home automated tele-management system. J Telemed Telecare 2018;24:410-9.

58 Finkelstein J, Lapshin O, Castro H, et al. Home-Based physical telerehabilitation in patients with multiple sclerosis: a pilot study. $J$ Rehabil Res Dev 2008;45:1361-73.

59 Paul L, Coulter EH, Miller L, et al. Web-Based physiotherapy for people moderately affected with multiple sclerosis; quantitative and qualitative data from a randomized, controlled pilot study. Clin Rehabil 2014;28:924-35.

60 Tallner A, Streber R, Hentschke C, et al. Internet-Supported physical exercise training for persons with multiple Sclerosis-A randomised, controlled study. Int J Mol Sci 2016;17:1667.

61 Donkers SJ, Nickel D, Paul L, et al. Adherence to PhysiotherapyGuided web-based exercise for persons with moderate-to-severe multiple sclerosis. Int J MS Care 2020;22:208-14.

62 Duncan E, O'Cathain A, Rousseau N, et al. Guidance for reporting intervention development studies in health research (guided): an evidence-based consensus study. BMJ Open 2020;10:e033516.

63 Bartholomew LK, Parcel GS, Kok G. Intervention mapping: a process for developing theory and evidence-based health education programs. Health Educ Behav 1998;25:545-63. 